\title{
BMJ Open A double masked randomised 4-week, placebo-controlled study in the USA, Thailand and Taiwan to compare the efficacy of oral valganciclovir and topical $2 \%$ ganciclovir in the treatment of cytomegalovirus anterior uveitis: study protocol
}

\author{
Jaskirat S Takhar, ${ }^{1,2}$ Ashlin S Joye, ${ }^{1,3}$ Thanapong Somkijrungroj, ${ }^{4,5}$ \\ Wipada Laovirojjanakul, ${ }^{6}$ Chang-Ping Lin, ${ }^{7}$ Thomas M Lietman, ${ }^{1,8}$ Travis C Porco, ${ }^{1,9}$ \\ Jeremy D Keenan (D) ,1,9 Elisabeth A Gebreegziabher (D) , ', Gerami D Seitzman, ${ }^{1,9}$ \\ Jennifer Rose-Nussbaumer, ${ }^{1,10}$ Thuy A Doan, ${ }^{1,9}$ Nisha R Acharya, ${ }^{1,9}$ \\ John A Gonzales ${ }^{1,9}$
}

To cite: Takhar JS, Joye AS, Somkijrungroj T, et al. A double masked randomised 4-week, placebo-controlled study in the USA, Thailand and Taiwan to compare the efficacy of oral valganciclovir and topical $2 \%$ ganciclovir in the treatment of cytomegalovirus anterior uveitis: study protocol. BMJ Open 2019;9:e033175. doi:10.1136/ bmjopen-2019-033175

- Prepublication history and additional material for this paper are available online. To view these files, please visit the journal online (http://dx.doi. org/10.1136/bmjopen-2019033175).

Received 23 July 2019

Revised 04 November 2019

Accepted 25 November 2019

Check for updates

(c) Author(s) (or their employer(s)) 2019. Re-use permitted under CC BY-NC. No commercial re-use. See rights and permissions. Published by BMJ.

For numbered affiliations see end of article.

Correspondence to Dr John A Gonzales; john.gonzales@ucsf.edu

\section{ABSTRACT}

Introduction Cytomegalovirus (CMV) anterior uveitis is a recognised cause of anterior uveitis in immunocompetent patients and is preventable cause of vision loss. Ocular sequelae include corneal endothelial damage which can cause corneal oedema and failure, as well as glaucoma. Recurrences of inflammation are common and therefore patients are often exposed to long-term therapy. Oral therapy is available in the form of valganciclovir, although with the caveat of systemic side effects such as bone marrow suppression and renal failure necessitating regular interval laboratory monitoring. Recent reports have demonstrated that topical $2 \%$ ganciclovir solution may offer promising treatment outcomes in patients with CMV anterior uveitis with superior safety, cost-effectiveness and convenience profiles. An investigation into the relative equipoise of these therapies is warranted for these reasons.

Methods and analysis The Systemic and Topical Control of Cytomegalovirus Anterior uveitis: Treatment Outcomes (STACCATO) trial is designed as a multicentre, block randomised by site, double-masked, placebo-controlled trial comparing the efficacy of oral valganciclovir, $2 \%$ topical ganciclovir and placebo in treating PCR-proven CMV anterior uveitis. Participant clinical evaluation will occur at three study time points by a masked study ophthalmologist over a 28-day period to assess resolution of ocular inflammation (secondary outcome). A control group will provide additional information about the possible impact that the infected host's immune response may play in controlling local viral replication. The primary analysis is an analysis of covariance (three arms) correcting for baseline to compare quantitative CMV viral load in the anterior chamber (AC) aqueous fluid before and 7 days after treatment.

\section{Strengths and limitations of this study}

- This randomised clinical trial compares both clinical and quantitative microbiological outcomes of cytomegalovirus treatment as opposed to solely clinical response.

- Baseline and end-line quantitative PCR viral loads from all sites that will be included in analysis are processed at the same laboratory, ensuring a uniform assessment of reduction in viral load.

- This is a multicentre clinical trial that includes three sites in Asia where the disease is most prevalent.

- Our limited sample size may not allow detection of the smallest meaningful difference between the treatments.

- Adherence is mainly assessed via self-report and may not accurately reflect treatment administration.

Ethics and dissemination The University of California San Francisco Committee on Human Research and the Khon Kaen University Institutional Review Board have given ethical approval. The results of this trial will be presented at local and international meetings and submitted for peerreviewed journals for publication.

Trial registration number NCT03576898.

\section{INTRODUCTION}

Cytomegalovirus (CMV) has been recognised as an important cause of posterior retinitis in patients with HIV/AIDS, particularly prior to the introduction of highly active antiretroviral therapy. However, CMV is now being implicated in immunocompetent individuals as a 
cause of anterior uveitis. ${ }^{1-4}$ Definitive diagnosis requires laboratory diagnosis, such as directed PCR. ${ }^{5}$

CMV anterior uveitis is a preventable cause of vision loss. It can cause damage to the corneal endothelial cells, which can lead to oedema, failure and, ultimately, requirement for corneal transplant. ${ }^{6-9} \mathrm{CMV}$ can recur in transplanted grafts, necessitating further surgeries and complications. ${ }^{10-14}$ Additionally, recurrences of inflammation in CMV anterior uveitis can be associated with glaucoma, which can cause irreversible blindness if not appropriately managed. ${ }^{15-17}$

While CMV anterior uveitis is typically characterised by recurrent bouts of inflammation, it is unknown whether each recurrence is mediated by active viral replication or a sterile immune response. Consequently, patients may be prescribed frequent oral or topical antiviral therapy. An understanding of the most effective treatment strategy for CMV anterior uveitis will help inform future studies investigating ways of decreasing recurrences of inflammation in this condition.

One option for the management of CMV anterior uveitis is oral valganciclovir $900 \mathrm{mg}$ two times per day. ${ }^{18} 19$ Prevention of recurrent inflammation in CMV anterior uveitis has been with valganciclovir $450 \mathrm{mg}$ two times per day. ${ }^{18} 19$ Oral valganciclovir can be associated with serious systemic side effects including renal failure and bone marrow suppression, necessitating regular interval laboratory monitoring. Topical ganciclovir $2 \%$ has also been used to treat and prevent recurrences of CMV anterior uveitis. ${ }^{20-22}$ Topical therapy is attractive as it does not require laboratory monitoring, although ocular side effects including corneal epitheliopathy and conjunctivitis could preclude long-term use. ${ }^{23} 24$ Thus, comparing oral valganciclovir $900 \mathrm{mg}$ two times per day to topical ganciclovir $2 \%$ six times per day has equipoise in the uveitis/cornea specialist.

To gain insight into preferred practice patterns for the treatment of CMV anterior uveitis, we surveyed attendees of the Biennial Chulalongkorn-Khon Kaen-Proctor-UCSF (CKPU) meeting in Bangkok, Thailand, on 5 March 2019. Survey questions were provided in a Likert scale, multiple-choice format with participants asked about their agreeability on seven statements. Answer choices varied from 1 to 4 ; 1 representing 'strongly disagree', 2 representing 'somewhat disagree', 3 representing 'somewhat agree' and 4 representing 'strongly agree'. Sixtyeight faculty level physicians responded to our survey, 5 practising in North America and 63 practising in Asia. Results are summarised in table 1. Physician preference for treatment medication of CMV anterior uveitis in presented in table 2. The results demonstrate that a consensus regarding the best treatment for CMV anterior uveitis is not yet established.

Some cases of CMV anterior uveitis have been noted to resolve without antiviral treatment. ${ }^{25}$ In this setting, having a trial in which oral valganciclovir is compared with topical ganciclovir, and including a control group where no antiviral was used would provide additional information about the possible impact that the infected host's immune response may play in controlling local viral replication.

\section{METHODS AND ANALYSIS \\ Study overview}

The Systemic and Topical Control of Cytomegalovirus Anterior uveitis: Treatment Outcomes (STACCATO) trial is a randomised, participant-masked and

Table 1 Survey results regarding CMV anterior uveitis practice patterns at the biennial CKPU meeting in Bangkok, Thailand $(n=68)$

\begin{tabular}{|c|c|c|c|c|}
\hline & $\begin{array}{l}\text { Strongly } \\
\text { disagree }\end{array}$ & $\begin{array}{l}\text { Somewhat } \\
\text { disagree }\end{array}$ & $\begin{array}{l}\text { Somewhat } \\
\text { agree }\end{array}$ & Strongly agree \\
\hline $\begin{array}{l}\text { 1. I think that oral valganciclovir is an effective medication to treat CMV } \\
\text { anterior uveitis }\end{array}$ & $0(0 \%)$ & 8 (11.8\%) & 36 (52.9\%) & 24 (35.3\%) \\
\hline $\begin{array}{l}\text { 2. I think that topical ganciclovir } 2 \% \text { eye drops is an effective medication } \\
\text { to treat } \mathrm{CMV} \text { anterior uveitis }\end{array}$ & $1(1.5 \%)$ & $11(16.2 \%)$ & 33 (48.5\%) & $23(33.82 \%)$ \\
\hline $\begin{array}{l}\text { 3. I think that oral valganciclovir is a safe medication to treat short term } \\
\text { ocular inflammation related to CMV anterior uveitis }\end{array}$ & $0(0 \%)$ & $11(16.2 \%)$ & 36 (52.9\%) & 21 (30.9\%) \\
\hline $\begin{array}{l}\text { 5. I think that topical ganciclovir eyedrops are a safe medication to treat } \\
\text { short-term ocular inflammation related to CMV anterior uveitis }\end{array}$ & $1(1.5 \%)$ & $8(11.8 \%)$ & $28(41.2 \%)$ & $31(45.6 \%)$ \\
\hline $\begin{array}{l}\text { 6. I think that topical ganciclovir eyedrops are a safe medication to treat } \\
\text { long-term ocular inflammation and prevent recurrences of CMV anterior } \\
\text { uveitis }\end{array}$ & $1(1.5 \%)$ & 17 (25.0\%) & 31 (45.6\%) & 19 (27.9\%) \\
\hline
\end{tabular}

CMV, cytomegalovirus. 
Table 2 Survey results regarding preferred treatment therapy of CMV anterior uveitis at the Biennial Chulalongkorn-Khon Kaen-Proctor-UCSF meeting in Bangkok, Thailand $(n=68)$

\begin{tabular}{|c|c|c|c|}
\hline & $\begin{array}{l}\text { Oral } \\
\text { valganciclovir }\end{array}$ & $\begin{array}{l}\text { Topical ganciclovir } \\
\text { eyedrops }\end{array}$ & No treatment \\
\hline $\begin{array}{l}\text { 1. The medication I am most likely to use to treat CMV anterior uveitis } \\
\text { is... }\end{array}$ & 25 (36.8\%) & 39 (57.4\%) & $4(5.88 \%)$ \\
\hline $\begin{array}{l}\text { 2. If cost were not an issue my preferred medication to treat CMV } \\
\text { anterior uveitis would be... }\end{array}$ & 39 (56.5\%) & 27 (39.1\%) & $1(1.5 \%)$ \\
\hline
\end{tabular}

CMV, cytomegalovirus.

ophthalmologist-masked, placebo-controlled trial comparing the efficacy of oral valganciclovir and $2 \%$ topical ganciclovir in treating PCR-proven CMV anterior uveitis. Study participants will be block-randomised with 1:1:1 allocation into the three study treatment arms. Participants will return to clinic for three study evaluations by a masked study ophthalmologist over a 28-day period at which point they will be assessed for resolution of ocular inflammation. All groups will receive their assigned treatment for 28 days. An objective measure of treatment efficacy will be assessed via biological samples of $\mathrm{AC}$ aqueous fluid before and 7 days after treatment. An overview of study procedures and study timeline is provided in table 3 . We used the Standard Protocol Items: Recommendations for Interventional Trials checklist when writing our protocol. ${ }^{26}$

\section{Specific aims and outcomes}

The specific aims of this trial are (1) to assess reduction in CMV viral load by treatment (as measured by $\log _{10}$-transformed quantitative viral load pretreatment compared with 7 days after treatment and (2) to assess the proportion of participants achieving clinical quiescence after 7 and 28 days of treatment. As a nested observational aim within our primary aim, we will evaluate the effect of topical corticosteroid started prior to enrolment on viral load yield by directed quantitative PCR. The research hypothesis for this study is that oral valganciclovir therapy will demonstrate the greatest efficacy in treating CMV anterior uveitis compared with $2 \%$ topical ganciclovir and placebo therapy.

Table 3 Timeline of major study procedures

\begin{tabular}{ll}
\hline Examination visit & Procedures \\
\hline Prestudy visit (exam \#0)- & Clinical eye examination suggests a viral aetiology \\
routine care appointment & AC paracentesis is performed to establish a viral aetiology \\
& - Half of fluid will be used for in-house directed PCR for HSV, VZV and CMV \\
& At least $50 \mu \mathrm{L}$ of fluid will be preserved and used for quantitative CMV PCR to be conducted \\
at a single US laboratory in eligible participants & Laboratory screening orders (CBC, chemistry panel, pregnancy test, HIV status) \\
& Patients will be prescribed topical corticosteroid (prednisolone acetate 1\%) to be used one \\
& drop in affected eye four times per day (this is typical standard of care) \\
& Patients requiring management of elevated IOP will be prescribed IOP-lowering medication \\
& according to treating ophthalmologist's discretion and best medical judgement \\
Exam \#1 (day 1 of study (7 & Review of results of in-house CMV PCR and laboratory results \\
days after exam \#0)) & Consent, enrolment, randomisation to treatment arm if participants meet eligibility criteria \\
& Treatment initiation \\
& Clinical eye examination (in the following order, VA, slit lamp examination of anterior segment, \\
& IOP) \\
& Endothelial cell morphology and density using specular microscopy or confocal microscopy \\
Exam \#2 (day 7 of study) & Clinical eye examination (in the following order, VA, slit lamp examination of anterior segment, \\
& IOP) \\
& AC paracentesis \#2 \\
Laboratory monitoring orders (CBC, Cr, BUN) & Clinical eye examination (in the following order, VA, slit lamp examination of anterior segment, \\
Exam \#3 (day 28 of study) & IOP) \\
& Endothelial cell density using specular microscopy or confocal microscopy \\
& Laboratory monitoring orders (CBC, Cr, BUN)
\end{tabular}

$\mathrm{AC}$, anterior chamber; BUN, blood urea nitrogen; CBC, complete blood count; CMV, cytomegalovirus; Cr, creatinine; HSV, herpes simplex virus; IOP, intraocular pressure; VA, visual acuity; VZV, varicella-zoster virus. 
Table 4 Study participant inclusion and exclusion criteria

\section{Inclusion criteria}

- CMV positivity by directed PCR from an anterior chamber paracentesis specimen conducted at any time in the past

- Active anterior uveitis using Standardisation of Uveitis Nomenclature (SUN) Working group with clinical impression of $\mathrm{CMV}$ as the etiologic agent

- $\geq 1+$ anterior chamber cell AND/OR

- Other signs consistent with active inflammation, such as elevated IOP, corneal oedema and/or active keratic precipitates (KPs)

- Participant willingness to use an acceptable method of contraception during the study period (ie, pharmacologic, barrier methods or abstinence).

\section{Exclusion criteria}

- Participants $<20$ years of age

- Inactive anterior uveitis

- Active intermediate or posterior inflammation (involvement of vitreous, choroid or retina)

- Participants who have received antiviral therapy $<14$ days prior to enrolment

- Participants who have received periocular or intraocular corticosteroid injection $<8$ weeks prior to enrolment

- Current use of oral corticosteroids

- Immunocompromised participants (primary or secondary immunodeficiency disorders)

- Prior immunosuppressive therapy in the past 3 months

- Directed PCR testing positive for HSV or VZV

- Plans to conceive during the study period, pregnant or breastfeeding mothers (blood or urine pregnancy test for all females of childbearing age is mandatory within 4 weeks prior to enrolment)

- Complete blood count with white blood cell, absolute neutrophil or platelet count lower than the lower limit of reference laboratory normal

- Blood urea nitrogen or creatinine above the upper limit of reference laboratory normal

- Recent ocular surgery within the past 30 days or planned surgery within the next 45 days

- Systemic autoimmune disease or ocular condition (besides anterior uveitis) anticipated to dictate or alter treatment course

CMV, cytomegalovirus; HSV, herpes simplex virus; VZV, varicella-zoster virus.

\section{Setting and eligibility}

The seroprevalence of CMV varies geographically. In East Asia, it estimated to be as high as $87 \%-100 \%$, whereas in Western countries, it is between $51.5 \%$ and $54.4 \% .^{27-29}$ For comparative purposes, four tertiary academic centres located in three countries will be included, one in the USA, two in Thailand and one in Taiwan.

Participants must provide written, informed consent before any study procedures can occur (see online supplementary appendix 1 for a sample informed consent). Study inclusion and exclusion criteria are outlined in table 4 .

\section{Recruitment}

Each study centre will screen subjects independently adhering to the inclusion and exclusion criteria outlined. Patients who are found to be CMV positive by in-house PCR and who meet eligibility criteria will be approached about participating in the study. Participants who have a history of previously diagnosed CMV anterior uveitis are also eligible to participate in the study. However, like other participants, their participation in the trial would require that they agree to an anterior chamber (AC) paracentesis to confirm that their current flare has demonstrable CMV present and to quantify the current flare's viral load to which it would be compared at 7 days post-randomisation.
Participant enrolment and project activities are planned to begin in October 2019 and end August 2021.

\section{Assignment of intervention}

Competitive block (of 3 and 6) randomisation of the participants, stratified by site will ensure that an equal number of participants at each site are randomised to study arms in a 1:1:1 ratio, not necessarily enrolling an equal number of participants at each site. Randomisation lists will be created by the coordinating centre clinical coordinator and accessed online via REDCap database. After consenting participants for enrolment and confirming all eligibility requirements have been met, the study coordinators who are masked to the allocation sequence assign the participants an ID from a predetermined list and will $\log$ into REDCap and perform the randomisation. The now unmasked study coordinators will then give participants their assigned treatment.

\section{Masking}

All study ophthalmologists and participants will remain masked to treatment assignment after enrolment. All participants will receive both pills and eye drops, and will not know which medications are active. Study doctors have no part in handling the medication bottles, all participants will be given dark bags to place and keep their bottles 
in throughout the trial. Additionally, participants will meet with the study coordinator first, before seeing their study doctor at follow-up visits to review the participants' medication calendars, and study coordinators will hold participant calendars and study bottles in their office for the entire visit. Before bringing participants to the study doctor, the study coordinator will remind participants not to disclose any information concerning their treatment to their study ophthalmologist or any other participants they may come across. If the participant is experiencing intolerable side effects, the study coordinator and participant will speak with the study doctor concerning discontinuation of treatment.

The UCSF pharmacy will be responsible for purchasing and distributing bottles of the medications labelled with NDC/lot number and expiration dates to the Proctor Foundation, and then medications will be distributed to all study sites. Study ID labels and dosing instructions will be provided to each site on separate label sheets. After enrolment and randomisation, each site's study coordinator will place the study ID label on the bottle, before dispensing the drug to the participant. If personnel are unmasked at any time, a protocol deviation form must be completed.

\section{Intervention}

Participants will initially present with anterior uveitis suspected to be due to a viral aetiology. Participants will receive a routine standard of care work-up for such a suspected aetiology, which will include an AC paracentesis. ${ }^{30}$ Half of this fluid $(\sim 50 \mu \mathrm{L})$ will be used for qualitative PCR to detect CMV, HSV and VZV at each respective site. The other half $(\sim 50 \mu \mathrm{L})$ will be stored at $-80^{\circ} \mathrm{C}$. Patients will have serologic testing for complete blood count, renal function, pregnancy status (in women of childbearing age) and HIV status.

In the event a participant is ineligible for study inclusion, the stored $50 \mu \mathrm{L}$ aliquot will be discarded. In eligible participants, this aliquot will undergo quantitative CMV PCR at a US laboratory, where laboratory personnel will be masked to participant identification, treatment assignment and site. This aliquot will serve as the baseline (prior to treatment randomisation) CMV viral load.

Eligible participants will remain masked and randomised by the unmasked study site coordinator to one of three study treatment arms. After randomisation, all participants will receive both tablets and topical eye drops. One-third of participants will receive oral valganciclovir tablets and placebo eye drops (balanced salt solution, BSS), one-third will receive topical ganciclovir $2 \%$ eye drops and placebo tablets, and one-third will receive placebo eye drops (BSS) and placebo tablets. Dosing will be four tablets daily ( $900 \mathrm{mg}$ po two times per day) for oral medication and six drops per day for topical.

After 7 days of treatment, therapy participants will return for clinical examination and repeat $\mathrm{AC}$ paracentesis. Approximately $80 \mu \mathrm{L}$ will be stored for eventual quantitative CMV PCR by the aforementioned US laboratory, providing a post-treatment viral load value. Approximately $20 \mu \mathrm{L}$ will be stored for future studies, provided a participant provides consent. In addition, corneal endothelium will be assessed using confocal or specular microscopy (depending on availability of the particular imaging modality at each centre) and will be used to measure endothelial cell morphology and density on day 1 and day 28.

Since it is standard of care to prescribe topical corticosteroids to reduce the severity of intraocular inflammation in participants with anterior uveitis, all participants (regardless of randomisation) will be prescribed topical prednisolone acetate $1 \%$ and instructed to apply one drop to the affected eye three times per day. Additionally, patients requiring management of elevated intraocular pressure (IOP) will be prescribed IOP-lowering medication according to treating ophthalmologist's discretion and best medical judgement.

Once participants have finished 28 days of treatment, including the final clinic visit and evaluation, they will have completed the study. The treating ophthalmologist will determine whether any continued treatment is necessary. Any continued treatment will be based on the treating ophthalmologist's clinical best clinical judgement.

\section{Harms and modifications}

Non-serious adverse events may include any unfavourable medical occurrences in participants who have ever received study medication, regardless of any causal relationship with treatment. This may include increased intraocular pressure $(>24 \mathrm{~mm} \mathrm{Hg})$, abnormal laboratory findings (rise in creatinine from $\geq 1.5$ to $<2 \mathrm{mg} / \mathrm{dL}$, reduction of white blood cell count to below $>1000$ to $<2500$ / $\mu \mathrm{L}$, platelet count from 20000 to 75000 , or haemoglobin level from $>6.5$ to $<9 \mathrm{~g} / \mathrm{dL}$ ), concurrent accident or illness, increase in the frequency and severity of a pre-existing condition, side effects intolerable to participants (gastrointestinal upset, nausea, vomiting, fatigue), or signs of corneal or conjunctival toxicity (keratitis or conjunctivitis). ${ }^{232431}$

If a non-serious adverse event in the form of a laboratory abnormality occurs, dose reductions of valganciclovir to one tablet $(450 \mathrm{mg})$ two times per day and topical drop frequency to three times per day will be made. Repeat laboratory testing should be ordered at 7 days of treatment with the reduced dose to monitor for resolution.

Serious adverse events (SAEs) include any medical occurrence that results in the following outcomes, or any other adverse event classified as severe: creatinine $\geq 20 \mathrm{mg} / \mathrm{L}$, leucocytes $\leq 10^{\wedge} 9 / \mathrm{L}$, platelets $<20 * 10^{\wedge} 9 / \mathrm{L}$, haemoglobin $<65 \mathrm{~g} / \mathrm{L}$, death, non-elective surgery or hospitalisation for any reason, myocardial infarction, stroke, any life-threatening event, cancer, seizure, congenital anomaly/birth defect, disability or permanent damage, required intervention to prevent permanent impairment/damage.

An unmasked medical monitor will evaluate the details of all SAEs via a protocol deviation form and can request 
additional clinical information and recommend additional tests. The medical monitor will determine whether the event is a true SAE and whether it is likely related to the study drug. The medical monitor will recommend continuing the study medication versus dose reduction versus discontinuation. Additionally, if the investigator thinks the SAE is related to the study drug, he/she may stop the study medication anytime (including prior to evaluation by the medical monitor). Further medical management will be at the discretion of the treating ophthalmologist and any consulting services. These participants should continue with scheduled study visits if possible.

All SAEs will require the completion of a protocol deviation form and will be reported to the local institutional review board (IRB) and UCSF IRB.

\section{Adherence}

Participants will be given a treatment diary in the form of a weekly calendar on which they will be asked to record adherence as well as reasons for missed doses. At each study visit, the study coordinator will review with the participant their treatment calendar, and document findings in the Medication Log in each participant file. Pill counting will also be done for an objective measure of adherence. Participants who miss a scheduled dose of medication will be instructed to skip that dose and continue with their next scheduled dose of tablets or eye drops.

\section{Concomitant care}

Study ophthalmologists may use other topical adjunctive medications such as IOP-lowering medications and cycloplegic agents. These should be documented on the study forms at each visit. The protocol does not allow for intravenous, or injection antiviral therapy, additional antivirals or surgery during the study period. If such therapies are deemed necessary, the protocol deviation form should be completed.

\section{Protocol deviation}

In rare cases, study ophthalmologists may determine that a deviation from protocol is necessary. This is at the discretion of the treating ophthalmologist and could be undertaken for a variety of reasons including rescue treatment and treatment discontinuation due to intolerability or safety. Subjects that experience deviations in treatment protocol will continue in their initially assigned treatment group and their data will be analysed accordingly.

\section{Retention}

Participants will be considered to have dropped out from the study only if they declare they are no longer interested in further participation and not willing to return for any study visits, or are deceased. If participants are not willing to return for any study visits, no further information will be collected.

Participants who stop the study treatment for reasons other than efficacy, tolerability and/or safety or miss study visits and do not respond to contact are considered non-compliant participants. Non-compliance will be noted and participants will continue their study treatment.

\section{Outcome assessments}

Viral load reduction

Primary measurement of efficacy of the three treatments arms will be a comparison of the $\log _{10}$-transformed quantitative viral load PCR at baseline versus after 7 days of therapy. This measure provides an objective measurement of treatment efficacy. We note a dearth of published data regarding CMV viral loads pretreatment and posttreatment. An endpoint of 7 days was chosen as a prior study found viral loads to be frequently undetectable after 14 days of therapy. ${ }^{32}$ All quantitative PCR measurements will be conducted at a single laboratory (masked to treatment and site) within the USA to ensure the same assay will be used on the provided samples, permitting direct comparison of quantitative viral load values.

\section{Effect of topical steroids on baseline viral load}

Participants will be queried about the frequency of their topical steroids use to measure the effect of topical corticosteroid use prior to eligibility screening on CMV viral load. We anticipate that the dosing of corticosteroids prior to presentation or referral will vary depending on the practices of the referring physician. More frequent dosing of topical corticosteroids may affect the amount of virus recovered from the $\mathrm{AC}$ perhaps due to local immunosuppression.

\section{Clinical response to therapy}

For the clinical secondary outcome, participants will be classified as 'quiescent' if they demonstrate on clinical examination: less than or equal to 0.5+ACcell AND resolution of other signs associated with active inflammation, including increased IOP, corneal oedema and/or active keratic precipitates (KPs). Since active inflammation can feature elevated IOP, if IOP is not controlled at a study visit, the participant will be considered as 'not quiescent'. This designation, however, does not preclude the participant from receiving additional IOP-lowering medication and the treating ophthalmologist may institute additional IOP-lowering medications as deemed necessary. In addition, a subjective measure of efficacy will be assessed by asking participants to estimate how many days after initiating therapy they felt improvement in their symptoms.

\section{Methods: data collection, management and monitoring}

Paper forms for each participant will be completed by study personnel in real time when the participant is being assessed. The forms will be reviewed and cross-checked for consistency and completeness by the study coordinator within 24 hours of completion. Study coordinators at all sites will scan data collection forms and send them electronically to the Data Coordinating Centre (DCC) within 10 days of the participant visit and retain hard copies in a secure location. The DCC is responsible for supervising data collection, data management, data quality control, data analysis, event adjudication, and 
training and certification of study site staff in the data management systems. All data will be entered into the official electronic research database, REDCap, within 10 days of receipt of completed forms.

Discrepancies and missing values will be assessed by the data entry manager and resolved by queries sent to the study coordinator and appropriate observers. A log file will preserve the date and time of any changes, together with who entered the changes.

\section{Data and Safety Monitoring Committee}

The Data Safety and Monitoring Committee (DSMC) has been established and is independent with experts from diverse fields including biostatistics, epidemiology and ophthalmology. Only after the DSMC reviews and approves the protocol will participants be enrolled. The DSMC monitors severe or unexpected events that threaten the safety of participants and oversees the data collected throughout the duration of the study. The DSMC is responsible for reviewing the results of the interim analysis and determining whether the trial should continue with or without modification.

\section{Patient and public involvement}

No patient was involved in the study.

\section{STATISTICAL ANALYSIS PLAN}

\section{Sample size}

Limited data from the available studies reporting detectable CMV viral loads pretreatment and post-treatment were available to guide our power calculations. We estimate a pretreatment viral load of $5.4 \log _{10} \mathrm{IU} /$ $\mathrm{mL}^{62032} 33 \quad(\mathrm{SD} \pm 1.00 \mathrm{log})$ and post-treatment viral load of $4.88 \log _{10} \mathrm{IU} / \mathrm{mL}^{632-34}(\mathrm{SD} \pm 0.95 \mathrm{log})$, with correlation of 0.7 between baseline and follow-up viral load. With a sample size of 33 participants per arm with estimated $10 \%$ total loss to follow-up, we anticipate at least $80 \%$ power to detect a $0.61 \mathrm{log}$ difference in viral load (based on pairwise analysis subject to an alpha of $0.05 / 3)$.

\section{Interim analysis}

An interim analysis of efficacy is performed one-third of the way through the trial, with alpha set at 0.001 . The DSMC reviews the unmasked interim analysis and makes recommendations on the continuation of the trial. No interim analysis for futility is performed. We anticipate $80 \%$ power to detect a difference of $1.7 \log$ units between any two arms.

\section{Specific aim 1}

The outcome variable is $\log _{10}$ viral load as measured by quantitative PCR at 7 days. The primary prespecified analysis is a permutation $p$ value based on the Fisher F statistic, derived from a linear model predicting the log viral load (primary outcome variable) as a function of the baseline log viral load and the treatment arm (dummy coded), that is, analysis of covariance (ANCOVA). Estimation will be conducted by ordinary least squares. Type I error rate (alpha) of 0.05 will be used.

Quantities of interest for analysis will be quantitative PCR data from AC paracentesis aqueous fluid collected at the prestudy visit for each participant enrolled in the study. The second quantity of interest for comparative purposes will be quantitative viral load derived from the analysis of aqueous fluid from the second AC paracentesis conducted on study day 7 (exam \#2). The outcome will be modelled as a linear function of baseline value and the treatment arm. Specifically, we will test the null hypothesis of a common intercept for all three treatment arms. We will also present the change scores for additional information and insight.

All quantitative PCR viral loads will be $\log _{10}$-transformed, with units converting from $\mathrm{IU} / \mathrm{mL}$ to $\log _{10} \mathrm{IU} / \mathrm{mL}$. The baseline quantitative PCR for each participant and treatment will be used as a predictor for quantitative PCR after 7 days of treatment.

The purpose of supplementary analyses reported in this section is to assess the role of statistical choices and data quality choices in shaping the result. A Kruskal-Wallis non-parametric one-way analysis of variance (ANOVA) (using change scores) will be an alternative consideration as a statistical analysis choice for the primary outcome. As a methodological analysis/sensitivity analysis, we will also conduct a Box-Cox transformation of our quantitative PCR $\log$ transformation.

If the results of the primary analysis are significant, then pairwise comparisons based on the Fisher least significant difference will be conducted ( $\mathrm{p}$ values derived by permutation). We will also test for the hypothesis of interaction between study country in the two Asian sites (Taiwan and Thailand). This will be an assessment of the heterogeneity of effect between study sites. In addition, we will repeat the primary analysis including an additional random effect for study site and report the estimated treatment effects under this supplemental model.

\section{Specific aim 2}

To compare the proportion of clinical quiescence at day 7 in the three treatment arms, a logistic regression model will be conducted. The outcome variable will be the binary classification of quiescence of inflammatory status determined by the masked study ophthalmologist. The analysis will be two-sided, with a type I error rate (alpha) of $0.05 / 2$; the predictor is the study arm (as a factor). In addition, we will repeat this analysis for data collected on day 28. We propose a Bonferroni correction, for an alpha of $0.05 / 2$. We will also tabulate the number of individuals who change status, reporting the $2 \times 2$ table of day 7 versus day 28 status, by arm. Missing data will be reported, and a sensitivity analysis will be conducted to assess the degree to which missing data could have affected the findings. Data collected at \pm 2 days will be used (day 7 data can be collected between days 5-9, and day 28 data can be collected at 26-30 days). 
Participants will also be administered a questionnaire querying when they felt symptomatic improvement other their condition. This analysis will be viewed as hypothesis generating and will be treated as supplementary and in addition to the main finding.

\section{Nested observational aim}

To assess the observational effect of topical corticosteroid administered prior to study enrolment on diagnostic yield of quantitative PCR, an ANCOVA of log-transformed mean viral load at initial AC paracentesis versus frequency of prestudy topical steroid use will be conducted. The analysis will be two-sided with a type I error rate (alpha) of 0.05 . We will report the findings unadjusted as hypothesis generating for a future larger trial. This will be sharply demarcated from the primary analysis. Bonferroni-Holm correction will be considered for this sub-aim.

\section{Secondary analyses}

In addition, an efficacy analysis will be conducted in which we include phenotype as a binary predictor (and the interaction with treatment). Phenotype of CMV anterior uveitis will be determined by the masked study ophthalmologist (acute/recurrent vs chronic). Analysis will consist of an ANCOVA as before, with the additional covariates. We will perform a hypothesis test of the coefficient for the phenotype-treatment interactions, two-sided, with a type I error rate (alpha) of 0.05 .

Individual level missing data (due to loss to follow-up or dropouts) is expected. Complete analysis will be reported. Missing values will be tabulated by treatment arm and study site. Sensitivity analysis assigning outcomes to the missing values will be reported. Additionally, analyses will be reported in which we adjust for any baseline covariate known to be a predictor of missing outcome data.

\section{ETHICS AND DISSEMINATION}

This protocol and informed consent forms have been approved by the IRB at the University of California, San Francisco and Khon Kaen University. The trial is registered at clinicaltrials.gov (NCT03576898). Protocol modifications are submitted to the relevant parties for review and/or approval. Subsequent to initial review and approval, the responsible local IRB/ethics committees (ECs) will review the protocol annually. The investigators will make safety and progress reports to the IRBs/ECs at least annually and within 3 months of study termination or completion at their site. These reports will include the total number of participants enrolled, adverse events and summaries of each DSMC review of safety and/or efficacy meeting. On completion of the 28-day study treatment

\begin{tabular}{|c|c|}
\hline Data category & Information \\
\hline Primary registry and trial identifying number & $\begin{array}{l}\text { ClinicalTrials.gov } \\
\text { NCT03576898 }\end{array}$ \\
\hline Date of registration in primary registry & 3 July 2018 \\
\hline \multicolumn{2}{|l|}{ Secondary identifying numbers } \\
\hline Contact for queries & John A. Gonzales, MD (john.gonzales@ucsf.edu) \\
\hline Title & $\begin{array}{l}\text { Systemic and Topical Antiviral Control of Cytomegalovirus Anterior uveitis: } \\
\text { Treatment Outcomes }\end{array}$ \\
\hline Countries of recruitment & Thailand, Taiwan, USA \\
\hline Health condition or problem studied & Cytomegalovirus anterior uveitis treatment \\
\hline Key eligibility criteria & $\begin{array}{l}\text { CMV positive anterior chamber paracentesis, active signs of anterior uveitis, no } \\
\text { history of immunodeficiency, normal laboratory values for } \mathrm{CBC} \text { and renal function }\end{array}$ \\
\hline Study type & Competitive block randomised, placebo controlled clinical trial \\
\hline Date of first enrolment & $\mathrm{N} / \mathrm{A}$ \\
\hline Target sample size & 99 to 33 per treatment arm \\
\hline Primary outcome & Change in log-transformed viral load \\
\hline Key secondary outcomes & $\begin{array}{l}\text { Objective and subjective time to achieve clinical quiescence, and the relationship } \\
\text { of topical steroid strength and dosing on initial anterior chamber paracentesis } \\
\text { quantitative viral load for CMV }\end{array}$ \\
\hline Project timeline & July 2019-August 2021 \\
\hline
\end{tabular}

$\mathrm{CMV}$, cytomegalovirus. 
protocol, the participant's treatment plan can be modified in any way the participant's ophthalmologist feels fit.

Written consent will be obtained for all study procedures after explaining the patient's disease, prognosis and treatment options, discussing the risks and benefits of participation, and addressing the patient's questions and concerns. The protocol site-specific informed consent forms in local language (Thai, Chinese or English), participant education and recruitment materials, other requested documented and any subsequent modifications shall also be reviewed and approved by the ethical review bodies at each respective site. Table 5 summarises the study protocol and trial registration information.

Only key study personnel will have access to identifying information of participants, while administrative forms and specimens will be deidentified.

A portion of biological samples from the second AC paracentesis (approximately $20 \mu \mathrm{L}$ of aqueous fluid) will be obtained for use in a future study of virus genome assay. A consent will be obtained and included in the main study consent form to specifically address the collection and future studies of such aqueous fluid.

The results of this trial will be presented at local and international meetings and submitted to peer-reviewed journals for publication.

\section{Author affiliations}

${ }^{1}$ Proctor Foundation, University of California San Francisco, San Francisco, California, USA

${ }^{2}$ John Burns School of Medicine, University of Hawaii, Honolulu, Hawaii, USA

${ }^{3}$ Touro University California College of Osteopathic Medicine, Vallejo, California, USA

${ }^{4}$ Ophthalmology, Chulalongkorn University Faculty of Medicine, Bangkok, Thailand

${ }^{5}$ Ophthalmology, King Chulalongkorn Memorial Hospital, Bangkok, Thailand

${ }^{6}$ Ophthalmology, Faculty of Medicine, Khon Kaen University, Khon Kaen, Thailand

${ }^{7}$ Ophthalmology, National Taiwan University Hospital, Taipei, Taiwan

${ }^{8}$ Department of Ophthalmology, University of California, San Francisco, California, USA

${ }^{9}$ Department of Epidemiology and Biostatistics, University of California, San Francisco, California, USA

${ }^{10}$ Kaiser Permanente, Redwood City, California, USA

Contributors JAG, TS, WL, TLM, ASJ and JST conceived of the study. JAG, TS, WL, TML, C-PL, JDK, GDS, JR-N, TAD, NRA, TCP, EAG, ASJ and JST initiated the study design and helped with implementation. JAG is the funding holder. TCP provided statistical expertise in clinical trial design. TCP and EAG will conduct the primary statistical analysis. JST, ASJ, TS, WL, C-PL, TML, TCP, JDK, EAG, GDS, JR-N, TAD, NRA and JAG contributed to refinement of the study protocol and approved the final manuscript.

Funding This work was supported by Huang Pacific Foundation and That Man May See Foundation. Sponsor: JAG at the Francis I. Proctor Foundation, University of California, San Francisco. The study sponsor was primarily involved in the study design; collection, management, analysis and interpretation of data; writing of the report and the decision to submit the report for publication. The study funders had no role in study design; collection, management, analysis or interpretation of data; writing of the report or the decision to submit the report for publication. Committees/Collaborators: John Gonzales (principal investigator); Nisha Acharya and Thuy Doan (Co-investigators and Editorial Committee); Gerami Seitzman (medical monitor); Thomas Lietman, Jennifer Rose-Nussbaumer, Jeremy Keenan, and Charles Lin (Data and Safety Monitoring Committee); Travis Porco (Biostatistician); Catherine Oldenberg (data coordinating centre supervisor); Sarah Lopez (study site coordinator); Ashlin Joye, Jaskirat Takhar, and Elisabeth Gebreegziabher (data managers and study coordinators); Thanapong Somkijrungroj (site director, King Chulalongkorn University); Wipada Laovirojjanakul (site director, Khon Kaen University); Chang-Ping Lin (site director,
National Taiwan University); Ruedee Kumklee (study coordinator, Khon Kaen University).

Competing interests None declared.

Patient consent for publication Not required.

Provenance and peer review Not commissioned; externally peer reviewed.

Open access This is an open access article distributed in accordance with the Creative Commons Attribution Non Commercial (CC BY-NC 4.0) license, which permits others to distribute, remix, adapt, build upon this work non-commercially, and license their derivative works on different terms, provided the original work is properly cited, appropriate credit is given, any changes made indicated, and the use is non-commercial. See: http://creativecommons.org/licenses/by-nc/4.0/.

\section{ORCID iDs}

Jeremy D Keenan http://orcid.org/0000-0002-7118-1457

Elisabeth A Gebreegziabher http://orcid.org/0000-0003-2454-5016

\section{REFERENCES}

1 Markomichelakis NN, Canakis C, Zafirakis P, et al. Cytomegalovirus as a cause of anterior uveitis with sectoral iris atrophy. Ophthalmology 2002;109:879-82.

2 Hwang Y-S, Shen C-R, Chang SHL, et al. The validity of clinical feature profiles for cytomegaloviral anterior segment infection. Graefes Arch Clin Exp Ophthalmol 2011;249:103-10.

3 Chee S-P, Jap A. Presumed Fuchs heterochromic iridocyclitis and Posner-Schlossman syndrome: comparison of cytomegaloviruspositive and negative eyes. Am J Ophthalmol 2008;146:883-9.

4 Oral acyclovir for herpes simplex virus eye disease: effect on prevention of epithelial keratitis and stromal keratitis. herpetic eye disease Study Group. Arch Ophthalmol 2000;118:1030-6.

5 McCann JD, Margolis TP, Wong MG, et al. A sensitive and specific polymerase chain reaction-based assay for the diagnosis of cytomegalovirus retinitis. Am J Ophthalmol 1995;120:219-26.

6 Chee S-P, Bacsal K, Jap A, et al. Clinical features of cytomegalovirus anterior uveitis in immunocompetent patients. Am J Ophthalmol 2008;145:834-40.

7 Chee S-P, Bacsal K, Jap A, et al. Corneal endotheliitis associated with evidence of cytomegalovirus infection. Ophthalmology 2007;114:798-803

8 Alfawaz A. Cytomegalovirus-Related corneal endotheliitis: a review article. Saudi J Ophthalmol 2013;27:47-9.

9 Koizumi N, Suzuki T, Uno T, et al. Cytomegalovirus as an etiologic factor in corneal endotheliitis. Ophthalmology 2008;115:292-7.

10 Ang M, Sng CCA, Chee S-P, et al. Outcomes of corneal transplantation for irreversible corneal decompensation secondary to corneal endotheliitis in Asian eyes. Am J Ophthalmol 2013;156:260-6.

11 Sonoyama H, Araki-Sasaki K, Osakabe Y, et al. Detection of cytomegalovirus DNA from cytomegalovirus corneal endotheliitis after penetrating keratoplasty. Cornea 2010;29:683-5.

12 Anshu A, Chee S-P, Mehta JS, et al. Cytomegalovirus endotheliitis in Descemet's stripping endothelial keratoplasty. Ophthalmology 2009;116:624-30.

13 Abou Shousha M, Yoo SH, Sayed MS, et al. In Vivo Characteristics of Corneal Endothelium/Descemet Membrane Complex for the Diagnosis of Corneal Graft Rejection. Am J Ophthalmol 2017;178:27-37.

14 Basilious A, Chew HF. Topical ganciclovir for prophylaxis of cytomegalovirus Endotheliitis in endothelial keratoplasty. Cornea 2019;38:120-2.

15 Accorinti M, Gilardi M, Pirraglia MP, et al. Cytomegalovirus anterior uveitis: long-term follow-up of immunocompetent patients. Graefes Arch Clin Exp Ophthalmol 2014;252:1817-24.

16 Choi JA, Kim J-E, Noh S-J, et al. Enhanced cytomegalovirus infection in human trabecular meshwork cells and its implication in glaucoma pathogenesis. Sci Rep 2017;7:43349.

17 Xi L, Zhang L, Fei W. Cytomegalovirus-Related uncontrolled glaucoma in an immunocompetent patient: a case report and systematic review. BMC Ophthalmol 2018;18:259.

18 Zandi S, Bodaghi B, Garweg JG. Review for disease of the year: treatment of viral anterior uveitis: a perspective. Ocul Immunol Inflamm 2018;26:1135-42.

19 Pleyer U, Chee S-P. Current aspects on the management of viral uveitis in immunocompetent individuals. OPTH 2015;9:1017-28.

$20 \mathrm{CC} \mathrm{S}, \mathrm{FR} \mathrm{H}$, Wang $\mathrm{TH}$, et al. Clinical outcomes in cytomegaloviruspositive Posner-Schlossman syndrome patients treated with topical ganciclovir therapy. Am J Ophthalmol 2014;158:1024-31. 
21 Wong JXH, Agrawal R, Wong EPY, et al. Efficacy and safety of topical ganciclovir in the management of cytomegalovirus (CMV)-related anterior uveitis. J Ophthalmic Inflamm Infect 2016;6:10.

22 CC S, Wang IJ, Chen WL, et al. Topical ganciclovir treatment in patients with cytomegalovirus endotheliitis receiving penetrating keratoplasty. Clin Exp Ophthalmol 2013;41:339-47.

23 Keorochana N, Choontanom R. Efficacy and safety of an extemporaneous preparation of $2 \%$ ganciclovir eye drops in CMV anterior uveitis. BMJ Open Ophth 2017;2:e000061.

24 Antoun J, Willermain F, Makhoul D, et al. Topical ganciclovir in cytomegalovirus anterior uveitis. J Ocul Pharmacol Ther 2017;33:313-8.

25 A CS J. Cytomegalovirus-Associated anterior segment inflammation. Expert Review of Ophthalmology 2011;5:517-28.

26 Chan A-W TJ, Altman DG, Laupacis A, et al. Statement: defining standard protocol items for clinical trials. Ann Intern Med 2013;2013:200-7.

27 Wong A, Tan KH, Tee CS, et al. Seroprevalence of cytomegalovirus, Toxoplasma and parvovirus in pregnancy. Singapore Med $\mathrm{J}$ 2000;41:151-5.
28 Taechowisan T, Sutthent R, Louisirirotchanakul S, et al. Immune status in congenital infections by torch agents in pregnant Thais. Asian Pac J Allergy Immunol 1997; 15:93-7.

29 Tookey PA, Ades AE, Peckham CS. Cytomegalovirus prevalence in pregnant women: the influence of parity. Arch Dis Child 1992;67:779-83.

30 Trivedi D, Denniston AKO, Murray PI. Safety profile of anterior chamber paracentesis performed at the slit lamp. Clin Exp Ophthalmol 2011;39:725-8.

31 Roche Pharmaceuticals. Valganciclovir package insert. Available: https://www.accessdata.fda.gov/drugsatfda_docs/label/2001/ 21304lbl.pdf

32 Kandori M, Inoue T, Takamatsu F, et al. Prevalence and features of keratitis with quantitative polymerase chain reaction positive for cytomegalovirus. Ophthalmology 2010;117:216-22.

33 Kawaguchi T, Sugita S, Shimizu N, et al. Kinetics of aqueous flare, intraocular pressure and virus-DNA copies in a patient with cytomegalovirus iridocyclitis without retinitis. Int Ophthalmol 2007;27:383-6.

34 Kandori M, Miyazaki D, Yakura K, et al. Relationship between the number of cytomegalovirus in anterior chamber and severity of anterior segment inflammation. Jpn J Ophthalmol 2013;57:497-502. 